\title{
Retroperitoneal cystic immature teratoma: A case report
}

\author{
JUNXIA LI $^{1 *}$, PEIYOU GONG ${ }^{2 *}$, FENGLI LIU ${ }^{2}$, PING SUN $^{1}$ and CHENGRONG WU ${ }^{1}$ \\ Departments of ${ }^{1}$ Oncology and ${ }^{2}$ Radiology, Yantai Yuhuangding Hospital, Yantai, Shandong 264000, P.R. China
}

Received September 5, 2014; Accepted May 7, 2015

DOI: $10.3892 / 01.2015 .3256$

\begin{abstract}
Retroperitoneal cystic immature teratoma (RCIT) is a rare disease. RCITs manifest as solid and cystic masses. In pathological sections, cysts of various sizes, with internal hemorrhage and necrosis, are observed. Components of all germ layer tissue are also observed, the majority of which is located within the endoderm. As the tumor contains undifferentiated immature tissue components, RCITs are also termed malignant teratomas. Immature teratomas grow rapidly, often invading adjacent tissue to cause serious symptoms, and transfer through the blood and lymph vessels, often resulting in glandular cancer. The present study reports the case of an infant with RCIT. The female patient, aged six months and six days, was hospitalized due to an abdominal mass. Physical examination revealed a large mass $(10 \times 8 \mathrm{~cm})$ below the xiphoid in the epigastrium. The mass, which ranged from the xiphoid to the umbilical region, was friable, and possessed a smooth surface, clear boundaries and poor activity, without tenderness. Upper abdominal computed tomography (CT) revealed a large, solid, cystic mass in the left, middle and lower retroperitoneum. The patient was admitted to the Yantai Yuhuangding Hospital for surgery. The pre-operative examination was improved following admission by documenting parameters that included the results from routine blood tests, bleeding and clotting times and cardiography. Retroperitoneal tumor resection was then performed. During resection, the tumor was found to originate from the retroperitoneum. As the tumor involved the gastric wall, a section of the gastric wall was resected, in addition to the tumor. The resection surface was yellow and friable. Pathological examination of tumor tissue sections revealed the involvement of immature nerves and mesenchymal components, confirming the diagnosis of a grade II immature teratoma. Subsequent to six months of outpatient follow-up, the patient had recovered well, without
\end{abstract}

Correspondence to: Dr Ping Sun or Dr Chengrong Wu, Department of Oncology, Yantai Yuhuangding Hospital, 20 Yuhuangdingdong Road, Yantai, Shandong 264000, P.R. China

E-mail: gpy_6681258@163.com

E-mail: 13356997292@163.com

*Contributed equally

Key words: teratoma, retroperitoneal, immature, surgical resection recurrence. RCIT is a clinically rare disease, and the present study adds to the current understanding of this rare condition in infants.

\section{Introduction}

Retroperitoneal cystic immature teratoma (RCIT), also termed malignant teratoma, is composed of immature tissue undergoing embryogenesis, the majority of which is constituted by neuroglia and neural tube-like structures. RCIT demonstrates pathological manifestations such as undifferentiated cells and increased cell mitosis (1). RCIT rarely occurs in children, accounting for $<1 \%$ of all childhood tumors (2). The tumor has malignant potential and recurrence is common. As RCIT exhibits a high-risk of developing into malignant teratoma, the treatment modalities for RCIT are similiar to those for malignant teratoma, which include surgical resection and combined treatment with chemotherapy and radiotherapy (3).

Malignant germinomas, including seminoma, dysgerminoma, embryonic carcinoma and endodermal sinus tumors, were historically termed malignant teratomas. However, these tumors are the result of cellular de-differentiation in various regions during the cellular movement from the yolk sac to gonads during embryonic development. Malignant germinomas are not usually classified into teratomas, as they possess no pathologically observable triploblastic structure (4). Imaging techniques, including ultrasonography, computed tomography (CT) and magnetic resonance imaging (MRI), are required for the diagnosis and surgical management of teratomas (5). On MRI, RCIT exhibits a pattern of mixed signal intensity and enhancement, which presents as uniformed hypodensity on T1 weighted imaging (WI) and hyperintensity on T2WI, with enhancement of the tumor capsule. On CT imaging, RCIT often exhibits heterogeneous density due to the presence of various well-differentiated components. Subsequent to confirmation of the diagnosis of RCIT, an early resection procedure must be performed. The tumor requires thorough resection to prevent the recurrence of the tumor from any remaining pluripotent cells.

\section{Case report}

The study was approved by the ethics committee of Yantai Yuhuangding Hospital (Yantai, China). A female aged six months and six days was admitted to the Yantai Yuhuangding Hospital (Yantai, Shandong, China) for 7 days in April 2014, due to an abdominal mass. A 12-cm mass was identified in the 


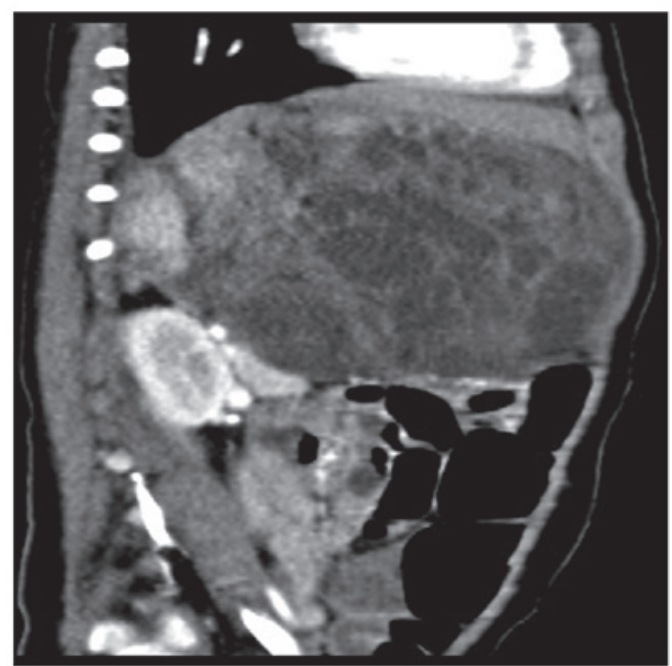

Figure 1. Computed tomography revealing low-density shadows that were observed in the retroperitoneal cystic mass, which was surrounded by enhanced separations.

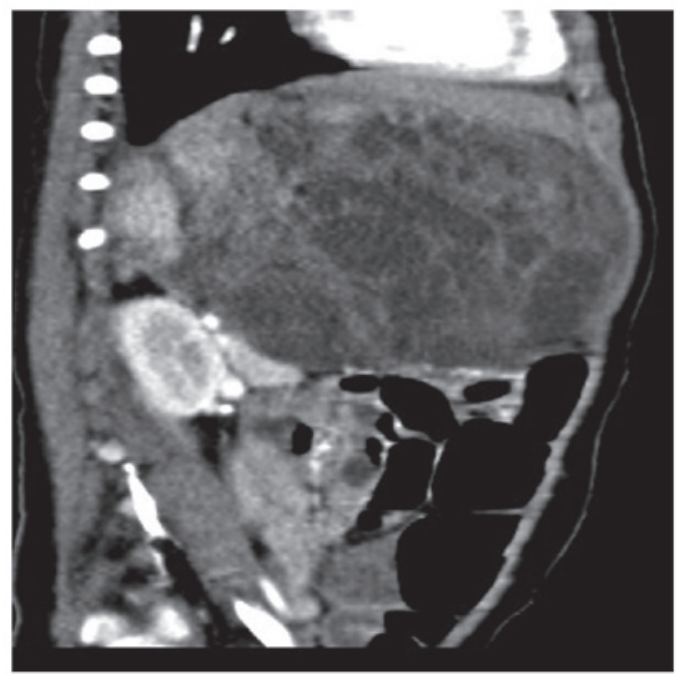

Figure 2. Sagittal view computed tomography revealing that the organs surrounding the tumor were pressed to produce shift.

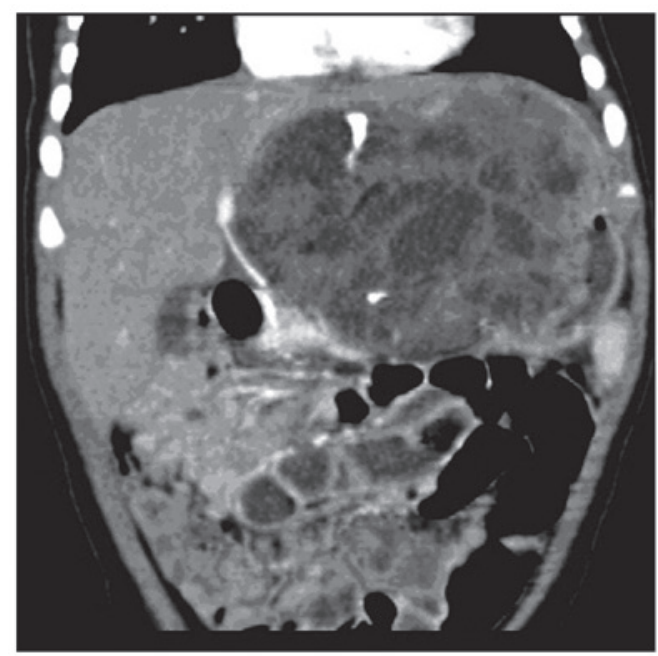

Figure 3. Coronal view of computed tomography, revealing plaque calcification.

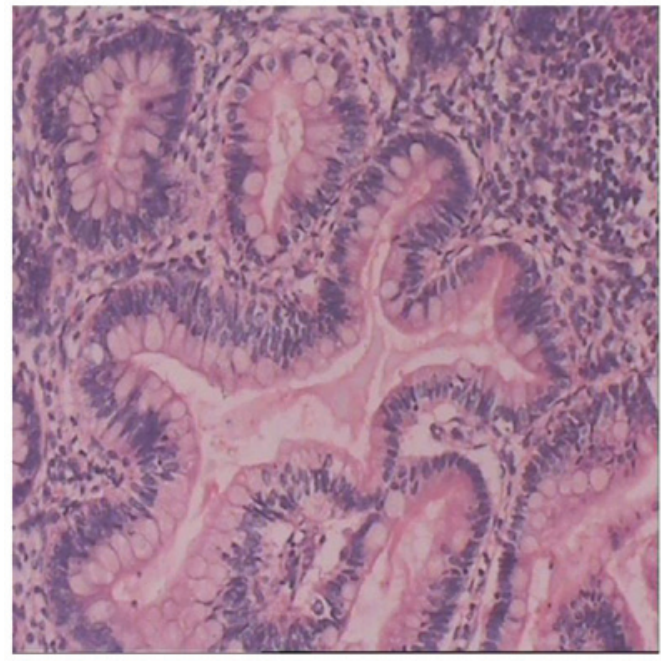

Figure 4. Tumor tissue was fixed with $4 \%$ neutral formaldehyde solution, dehydrated and embedded in paraffin. Tissue sections were sliced into $4-\mu \mathrm{m}$ thick microscopic sections. Morphological features of the tumor were assessed by hematoxylin and eosin staining. The pathological analysis of the tumor tissue revealed a small-to-moderate proportion of immature neural and mesenchymal components, harboring characteristics of a grade II immature teratoma (magnification, x100), and the cells formed a structure that simulated a Homer-Wright rosette.

abdomen of the infant one week prior to hospitalization. The infant demonstrated no abdominal distension, abdominal pain, diarrhea or fever. The patient was in high spirits, slept well and had a good appetite. No evident abnormalities or significant weight loss were observed.

The results of the physical examination revealed the presence of abdominal distention and clear abdominal veins. No evident dilation or bowel obstruction was identified. A large mass, $10 \times 8 \mathrm{~cm}$ in size, was found below the xiphoid in the epigastrium. The mass ranged between the xiphoid and umbilical region. The lesion was friable, and possessed a smooth surface, clear boundaries and poor activity, without tenderness or rebound tenderness. The liver, spleen and lower ribs were not examined. Bowel sounds were identified, without hyperthyroidism and gurgling.

Dynamic enhanced CT of the upper abdomen revealed a large solid cystic mass in the fat space of the left, middle and lower retroperitoneum. The internal diameter of the mass was indicated to measure $66 \times 100 \mathrm{~mm}$ by $\mathrm{CT}$, and the mass contained plaque calcification. Following enhanced scanning, the solid region of the tumor demonstrated evident enhancement and was shifted upon compression near the intestine, pancreas and left lobe of the liver (Figs. 1-3). The disease was likely to be diagnosed as a left, middle and lower retroperitoneal malignant tumor based on the imaging data. Retroperitoneal tumor resection was subsequently performed. During resection, the tumor was found to originate from the retroperitoneal space. As the tumor involved the gastric wall and grew into the gastric cavity, a section of the gastric wall was resected in addition to the tumor. The pathologically examined tumor tissue indicated the presence of nodosities. The resection surface was yellow and friable, with a portion that appeared similar to rotten meat and contained liquids and mucous. The tumor demonstrated infiltrative growth into 
the gastric wall, and did not involve the resection margins. A moderate quantity of immature nerves and mesenchymal components were evident in the microscopic examination of the tumor, which was diagnosed by pathology as grade II immature teratoma (Fig. 4). Except the routine prevention of infection and nutritional support treatment, no further treatment following surgery was required. Subsequent to six months of outpatient follow-ups, the patient had recovered well, without recurrence.

\section{Discussion}

Immature teratomas contain varying quantities of neuroectodermal or blastemal tissues and may be graded by the quantity of immature neuroglial tissue (6). Immature teratomas have been mostly reported in the ovaries of young females, and the diagnosis of these tumors is based mainly on the pathological evaluation of the tumor tissue. A previous immunohistological study investigating immature teratoma tissue reported the presence of partial neuroendocrine differentiation of immature origin (7). Complete resection, along with chemotherapy, is the main treatment method for immature teratomas (8).

Lee et al (9) reported immature teratoma of the parapharyngeal space, and reported that germ cell tumors often occur in infants, with the sacrococcygeal region, gonads and mediastinum as the most common sites. According to Anilkumar et al (10), gastric teratomas are extremely rare, accounting for $<1 \%$ of all teratomas in infants and children. In total, $>100$ cases of gastric teratomas have been reported in the literature, and extremely few of these are of the immature variety.

The present study reports the case of an infant that developed RCIT. The tumor involved the gastric wall, and was large, $\sim 12 \mathrm{~cm}$, in size. The clinical feature of the lesion was a progressively increasing painless mass. Imaging features included the identification of a cystoid mass surrounded by separations. Enhancement of the separations was evident in the mass, with calcification also observed. No clear fat components were identified in the lesion. The patient was diagnosed by post-operative pathology and immunohistochemical analysis. Immature neural tissues of various proportions, including primitive neural canal and neural epithelial components, and other immature components, including immature cartilage, primary small cells and mesenchymal tissues, were observed.

In summary, retroperitoneal immature teratoma is rare in clinical practice, and is challenging to diagnose. It is also difficult to differentiate retroperitoneal immature teratoma from retroperitoneal neurogenic and yolk sac tumors, which occurs mostly in the axis of the body. The present study provides additional information on the occurrence of this rare tumor in infants.

\section{Acknowledgements}

The authors thank the patient in the present study for their participation and colleagues in the Department of Pediatric Surgery of Yantai Yuhuangding Hospital for their assistance.

\section{References}

1. Aggarwal SK, Keshri A and Agarwal P: Immature teratoma of the nose and paranasal sinuses masquerading as bilateral nasal polyposis: a unique presentation. J Postgrad Med 59: 138-141, 2013.

2. Ishida M, Hotta M, Ohta M, Taga T, Ohta S, Takeuchi Y and Okabe $\mathrm{H}$ : A case of retroperitoneal immature teratoma with nephroblastic components. J Pediatr Hematol Oncol 34: e22-e25, 2012.

3. Yanai H, Matsuura H, Kawasaki M, Takada Y, Tabuchi Y and Yoshino Tadashi: Immature teratoma of the ovary with a minor rhabdomyosarcomatous component and fatal rhabdomyosarcomatous metastases: The first case in a child. Int J Gynecol Pathol 21: 82-85, 2002.

4. GrammatikopoulouI,KontomanolisEN,ChatzakiE,ChouridouE, Pavlidis P, Papadopoulos EM and Lambropouloux M: Immature malignant sacrococcygeal teratoma: case report and review of the literature. Clin Exp Obstet Gynecol 40: 437-439, 2013.

5. Hasiotou M, Vakaki M, Pitsoulakis G, Zarifi M, Sammouti H, Konstadinidou CV and Koudoumnakis E: Congenital cervical teratomas. Int J Pediatr Otorhinolaryngol 68: 1133-1139, 2004.

6. Marina NM, Cushing B, Giller R, Cohen L, Lauer SJ, Ablin A, Weetman R, Cullen J, Rogers P, Vinocur C, et al: Complete surgical excision is effective treatment for children with immature teratomas with or without malignant elements: A Pediatric Oncology Group/Children's Cancer Group Intergroup Study. J Clin Oncol 17: 2137-2143, 1999.

7. Bogner G, Wolfrum-Ristau P, Schneider W, Fischer T and Jacobs VR: Local foreign body reaction of peritoneum after rupture of cystic partially immature teratoma. J Minim Invasive Gynecol 21: 959-962, 2014.

8. Matsushita $\mathrm{H}$ and Tani $\mathrm{H}$ : Successful infertility treatment following fertility-sparing surgery and chemotherapy for ovarian immature teratoma: A case report and a literature review. Reprod Med Biol 10: 193-198, 2011.

9. Lee DH, Yoon TM, Lim SC and Lee JK: Immature teratoma of the parapharyngeal space presenting with airway obstruction in an infant. B-ENT 10: 71-73, 2014.

10. Anilkumar MG, Jagadishkumar K, Girish GN and Sunila: Immature gastric teratoma in an infant. Indian J Surg 75 (Suppl 1): 453-455, 2013. 